\title{
High-dose 3-dimensional conformal radiotherapy with concomitant vinorelbine plus carboplatin in patients with non-small cell lung cancer: A feasibility study
}

\author{
QIANG LIN ${ }^{1}$, JUN WANG ${ }^{2}$, YUE'E LIU ${ }^{1}$, HUILING SU ${ }^{1}$, NA WANG $^{1}$, YUEHUA HUANG ${ }^{1}$, \\ CHAO-XING LIU ${ }^{3}$, PING ZHANG ${ }^{2}$, YANNAN ZHAO ${ }^{1}$ and $\mathrm{KUN} \mathrm{CHEN}^{1}$
}

${ }^{1}$ Department of Oncology, Hebei Medical University Affiliated North China Petroleum Bureau General Hospital,
Renqiu, Hebei 062552; ${ }^{2}$ Department of Radiation Oncology, Hebei Medical University Fourth Hospital,
Shijiazhuang 050011; ${ }^{3}$ Department of Radiation Oncology, Beijing University First Hospital, Beijing10034, P.R. China

Received January 26, 2011; Accepted May 6, 2011

DOI: $10.3892 / \mathrm{ol} .2011 .317$

\begin{abstract}
The aim of this study was to evaluate the feasibility of high-dose 3-dimensional conformal radiotherapy (3DCRT) (70 Gy) with concomitant vinorelbine (NVB) plus carboplatin (CBP) chemotherapy in patients with non-small cell lung cancer (NSCLC). Patients with advanced NSCLC were treated with 3-dimensional conformal radiotherapy in conventional fractionation: $2 \mathrm{~Gy} /$ fraction, 1 fraction/day, 5 fractions/ week; total dose $70 \mathrm{~Gy}$. The radiotherapy planning of every case had met the following conditions: the percentage of total lung volume receiving 20 Gy (V20) $\leq 30 \%$ and the percentage of total lung volume receiving $30 \mathrm{~Gy}(\mathrm{~V} 30) \leq 20 \%$. Chemotherapy was commenced on the first day of radiotherapy: NVB $25 \mathrm{mg} / \mathrm{m}^{2}$, day 1 and day $8, \mathrm{CBP}$ at AUC of $5 \mathrm{mg} / \mathrm{ml}^{-1} \cdot \mathrm{min}^{-1}$, day 8 , repeated for 28 days, two concomitant cycles during radiotherapy, and not more than 4 cycles following radiotherapy. A total of 37 patients were recruited and each of them completed the entire radiation procedure. No Grade $\mathrm{V}$ toxicity was observed within the group. The hematological toxicity rates were: Grade III/IV neutropenia was observed in 18.9\% (7/37) of cases, Grade III/IV thrombocytopenia in $8.1 \%$ (3/37) of cases, but no cases of Grade III/IV anemia were noted. For non-hematological toxicities the rates were: Grade III radiation pneumonitis, $8.1 \%$ (3/37) of cases; Grade III radiation esophagitis, $13.5 \%$ (5/37); but no cases of Grade IV/V non-hematological toxicities. High-dose 3DCRT also achieved a favorable efficacy: the complete response (CR) rate was $13.5 \%(5 / 37)$ and the partial response (PR)
\end{abstract}

Correspondence to: Dr Qiang Lin, Department of Oncology, Hebei Medical University Affiliated North China Petroleum Bureau General Hospital, 8 Huizhan Avenue, Renqiu, Hebei 062552, P.R. China

E-mail: linqiang@csco.org.cn

Key words: non-small cell lung cancer, three-dimensional conformal radiotherapy, vinorelbine, carboplatin, concomitant radiochemotherapy rate was $64.9 \%(24 / 37)$. The total response $(\mathrm{CR}+\mathrm{PR})$ rate was $78.4 \%$ (29/37). The median survival time was 12 months and the 1 -year overall survival rate was $45.1 \%$. Given that $35 \%$ of patients in the study had stage IV disease, the survival results were comparable with other similar studies. In conclusion, in our small-sample exploratory study, the high-dose regimen of 70 Gy using 3DCRT with concomitant NVB plus CBP was feasible for patients with NSCLC. Further evaluation of this regimen is ongoing in a prospective controlled phase II trial.

\section{Introduction}

Lung cancer has the highest incidence and mortality rate of all malignancies. Non-small cell lung cancer (NSCLC) accounts for approximately $80 \%$ of all lung cancers, the majority of which are at an advanced stage and unresectable when diagnosed (1). As the main approach for NSCLC, radiotherapy was adopted exclusively until the 1990s when the advantage of radiotherapy combined with chemotherapy was established by CALGB8433 (2). In addition, Furuse et al concluded the superiority of the concomitant combination over the sequencing one (3), a theory that was supported by a recent meta-analysis (4). Nine to eleven months of the median survival time (MST) for patients with advanced NSCLC treated by radiotherapy alone has been improved to approximately 16 months for those treated with radiotherapy combined with chemotherapy (2-4). A total of 60 Gy was considered to be the standard dose in conventional fractionation radiotherapy, but only a 5-year survival rate of approximately $5 \%$ has been achieved since its clinical application (5). A higher radiation dose is required to improve the local control rate of tumors, and it has been suggested in the US RTOG (Radiation Therapy Oncology Group) trials that the radiation dose in the concomitant radiochemotherapy may be safely escalated to 70-74 Gy for 3-dimensional conformal radiotherapy (3DCRT) $(6,7)$. However, due to the absence of reports on high-dose tolerance of concomitant radiochemotherapy in Chinese patients with NSCLC, a prospective trial was required to assess the safety of high-dose radiation. One of the key factors that limited dose escalation was pulmonary volume radiation dose. The decrease in radiation dose of 
Table I. Treatment plan.

Concomitant radiochemotherapy regimen

RT regimen: Week 1-7: 2 Gy/f, 1 f/d, 5 f/w. Total dose: 70 Gy.

\begin{tabular}{|c|c|c|c|c|c|c|}
\hline Week & 1 & 2 & 3 & 4 & 5 & 6 \\
\hline $\mathrm{RT}$ & |IIII & |IIII & |IIII & |IIII & |IIII & |IIII \\
\hline
\end{tabular}

Chemotherapy: NVB $\left(25 \mathrm{mg} / \mathrm{m}^{2}\right)$ day 1 and day 8; CBP at AUC of $5 \mathrm{mg} / \mathrm{ml}^{-1} \cdot \mathrm{min}^{-1}$ on day 8 , repeated every 28 days. NVB CBP

CBP, carboplatin.

the two lungs made it possible to escalate the dose to 70 Gy in NSCLC. In our prospective small-sample exploratory study, 3DCRT was used to limit pulmonary radiation dose through a pulmonary dose-volume histogram (DVH) to treat NSCLC patients with 70 Gy high-dose radiation combined with concomitant NC chemotherapy [vinorelbine (NVB) plus carboplatin (CBP)] under the conditions of $\mathrm{V} 20 \leq 30 \%$ and $\mathrm{V} 30 \leq 20 \%$, based on previous reports $(8,9,10)$. The primary endpoint was to evaluate the tolerance of this regimen, and observe its preliminary efficacy.

\section{Patients and methods}

Patient characteristics. Between February 2008 and January 2010, 37 sequential untreated patients with pathologically or cytologically confirmed NSCLC were treated with high-dose 3DCRT with concomitant NC chemotherapy. The 37 patients comprised 22 males and 15 females aged between 42 and 70 years with a median age of 64 .

Eligibility. Hospitalized patients (aged $\geq 18$ and $\leq 70$ years) diagnosed pathologically or cytologically as stage III or IV (UICC1997) were recruited into the trial. Patients had at least one evaluable lesion, an expected survival time of $>3$ months and no other severe internal diseases that required hospitalization. Patients were requested to have a Karnofsky performance status of $\geq 60$. The required laboratory tests included a neutrophil count of $\geq 2.0 \times 10^{9} / 1$, a platelet count of $\geq 100 \times 10^{9} / 1$, a hemoglobin count of $\geq 100 \mathrm{~g} / 1$, and serum creatinine, aspartate aminotransferase, alanine aminotransferase, and total serum bilirubin supper limits of normal. The exclusion criteria were: cases at stage IIIb with effusion; cases with superior vena cava syndrome; pregnancy; lactation; a history of other malignancies, with the exception of carcinoma in situ of the cervix, non-melanomatous skin cancer, or cancer from which the patient had not been disease-free for five years; a general medical condition preventing combined modality therapy; and a known hypersensitivity to NVB or CBP; as well as any use of concurrent other antineoplastic therapy.

Pretreatment evaluation. Pretreatment evaluation included medical history, complete physical examination, chest and abdominal helical computed tomography (CT) scan, electrocardiography, bronchoscopy, bone marrow scan (if clinically indicated), complete blood count, and a biochemical profile. These pretreatment tests were performed in the week prior to treatment initiation. Patients received physical examinations, and blood counts were obtained once a week or more often if deemed necessary. A biochemical profile was obtained and electrocardiography was performed prior to each chemotherapy cycle.

Ethics. The procedures were approved by the Ethics Committee of Hebei Medical University and were performed in accordance with the ethical standards of human experimentation, and the Helsinki Declaration of 1975, as revised in 2000. All of the patients provided written informed consent.

Recruitment and treatment plan. Patients were prospectively recruited and treated with concomitant radiochemotherapy. The treatment scheme is shown in Table I. For all eligible patients, radiotherapy began on day 1 , concurrently with the first cycle of chemotherapy.

Radiotherapy. The patient was placed in a supine position with hands on head and fingers interlocked. A vacuum pad was used to fix the body position and appropriately limit respiratory movement. A contrast spiral CT (GE LightSpeed Plus 4) was performed, and the image data was input into the 3D therapeutic planning system. Venus 5014 software (Shanghai Tuoneng Co., Shanghai, China) was used to design the radiation plan. Delineation of the target volume was based on the consensus guidelines for the delineation of the radiotherapy target volume in NSCLC (11): the target of the primary lesion was delineated in the pulmonary window $(1600,-600 \mathrm{HU})$, and that of mediastinal lymph nodes was delineated in the mediastinal window (400, -20 HU). An involved field radiation was adopted, without prophylactic radiation on the lymph drainage field. The definition of the target volume was as follows: gross tumor volume (GTV) was defined as the primary lesion and the lymph node $>1 \mathrm{~cm}$ in the short diameter, clinical target volume (CTV) as the GTV enlarged by $6 \mathrm{~mm}$ for squamous cell carcinoma, large cell cancer and metastatic lymph nodes or by $8 \mathrm{~mm}$ for adenocarcinoma; and PTV as the CTV enlarged by $10-15 \mathrm{~mm}$ based on 
the respiratory movement observed under the X-ray simulator. The definition of GTV was confirmed jointly by a diagnostic imaging physician, a radiologist and a medical physicist. The outlines of vital organs and body surface were drawn by a radiologist. Three to six coplanar or non-coplanar fields were adopted for the conformal radiation. The therapeutic plan was optimized by DVH. A total of $100 \%$ of the isodose curve should cover at least $95 \%$ of the PTV. The restrictive conditions of the key organs included: V20 $\leq 30 \%$ and V30 $\leq 20 \%$ for the two lungs, $0 \%>45$ Gy for spinal marrow, esophageal length under 60 Gy radiation $\leq 10 \mathrm{~cm}$ and $0 \%>70 \mathrm{~Gy}$, and V40 $\leq 40$ Gy for heart. All of the cases were irradiated by a Siemens Premus Plus linear accelerator of 6MV-X, which was equipped with a 27-pair multileaf collimator (Topslane, Shanghai Tuoneng Co.). CT scans were repeated after irradiation dose (DT) reached 50 Gy. The radiation was continued as planned until completion unless an obvious shrinkage of the target volume was observed, for which a field reduction was necessary based on another contrast CT scan in the original body position. The lesions were involved in the same target field as far as possible, and a second field was defined if there was a large margin between lesions. Each of the patients was radiated for DT $70 \mathrm{~Gy}, 2$ Gy/fraction, once per day, 5 days per week for 7 weeks. X-ray combined with electron at a dose of DT 60-70 Gy was adopted in the case of supraclavicular nodular metastasis.

Chemotherapy. Chemotherapy was started on day 1 of radiation, with NVB $25 \mathrm{mg} / \mathrm{m}^{2}$ on days 1 and 8 intravenously, and $\mathrm{CBP}$ at AUC of $5 \mathrm{mg} / \mathrm{ml}^{-1} \cdot \mathrm{min}^{-1}$ on day 8 , which was repeated every 28 days. Two cycles of concomitant chemotherapy were carried out during radiotherapy, and another 4 cycles at most were carried out after the radiation. Antiemetic, white blood cell/platelet promotion and support care were used for each patient as required.

Evaluation criteria of adverse events and short-term treatment efficacy. NCI common toxicity criteria 2.0 (NCI CTC 2.0) were used to grade the toxicity (12). The short-term response was evaluated 4 weeks after the completion of radiotherapy, based on the objective criteria by WHO (13): complete response (CR): disappearance of all observable lesions, maintained for $>4$ weeks; partial response (PR): a decrease of at least $50 \%$ in the largest diameter or the product of the largest two vertical diameters, maintained for $>4$ weeks; stable disease (SD): a shrinkage of the product of two diameters, or $<50 \%$ or an increase of the product $\leq 25 \%$; progressive disease (PD): an increase of $>25 \%$ in at least one lesion, or appearance of new lesions. CR and PR were considered as a response, while SD and $\mathrm{PD}$ were considered as no response.

Dose attenuation. Dose modifications were based on the most serious toxicities that occurred on any day after the treatment plan commenced.

The irradiation dose was not allowed to be modified. However, radiotherapy was withheld for Grade III or higher toxicities until they were no longer present. Radiotherapy was continued, but chemotherapy was withheld in cases where Grade III or higher toxicities, unrelated to radiotherapy, occurred, such as peripheral neuritis. Chemotherapy was resumed when those toxicities were eliminated.
Chemotherapeutic doses were modified in the manner mentioned below. If Grade III-IV thrombocytopenia, Grade III-IV anemia, Grade IV neutropenia, or Grade III-IV non-hematological toxicity occurred (with the exception of Grade III nausea, vomiting and anorexia), both RT and NVB with CBP were withheld until the Grade III or IV toxicities were no longer present. If the toxicities were not eliminated within 2 weeks, the patient was withdrawn from the study. The NVB and CBP doses of the following chemotherapy cycle were reduced by $25 \%$. Prophylactic recombinant human granulocyte colony-stimulating factor was used following that chemotherapy cycle. If Grade III neutropenia or Grade II thrombocytopenia alone occurred, chemotherapy was stopped and radiotherapy continued. The NVB and CBP doses of the following chemotherapy cycle were the same as those in the original regimen. Prophylactic recombinant human granulocyte colony-stimulating factor was used following that chemotherapy cycle.

Subsequent treatment. A second-line regimen based on docetaxel was used in the case of PD with consideration for the general status of the patient.

Follow-up. Following treatment, patients were followed up at every 3 months for the first year, every 6 months for the second year, and annually thereafter. Each follow-up included history, physical examination, complete blood count, blood biochemical examination and chest CT.

Death from any cause was calculated from the date of treatment until the patient succumbed or until the last followup evaluation.

Statistical analysis. Statistical analysis was performed using the SPSS13.0 software package. The accumulated overall survival rate was calculated using the Kaplan-Meier method.

\section{Results}

Patient characteristics. A total of 37 sequential untreated patients with pathologically or cytologically confirmed NSCLC were treated with high-dose 3DCRT with concomitant NC chemotherapy. The 37 patients included 22 males and 15 females, aged between 42 and 70 years, with a median age of 64 . Of the 37 patients, 20 patients had squamous cell carcinoma, 16 had adenocarcinoma and one had large cell cancer. Seven had stage IIIa disease, 17 had stage IIIb disease and 13 had stage IV disease.

The patients were followed up until they succumbed to the disease or until the time of the last follow-up evaluation. Until May 31, 2010, no patients were lost to follow-up. The median follow-up time for all patients was 12 months (range 4-16).

Treatment compliance. All 37 patients completed the 70 Gy radiotherapy and at least 2 cycles of concomitant NC chemotherapy. Toxicity and response were evaluated in all 37 patients.

Adverse events. No treatment-associated death (Grade V toxicity) occurred in any of the patients. 
Table II. Hematological toxicities of concomitant radiochemotherapy.

\begin{tabular}{|c|c|c|c|c|c|c|}
\hline & 0 & I & II & III & IV & Total \\
\hline & Cases (\%) & Cases $(\%)$ & Cases $(\%)$ & Cases $(\%)$ & Cases (\%) & Cases (\%) \\
\hline Neutropenia & $5(13.5)$ & $9(24.3)$ & $16(43.2)$ & $5(13.5)$ & $2(5.4)$ & $32(86.5)$ \\
\hline Thrombocytopenia & $20(54.1)$ & $10(27.0)$ & $4(10.8)$ & $2(5.4)$ & $1(2.7)$ & $17(45.9)$ \\
\hline Anemia & 25 (67.6) & $6(16.2)$ & $6(16.2)$ & $\begin{array}{ll}0 & (0)\end{array}$ & $0 \quad(0)$ & $12(32.4)$ \\
\hline
\end{tabular}

Table III. Non-hematological toxicities of concomitant radiochemotherapy.

\begin{tabular}{|c|c|c|c|c|c|c|}
\hline & 0 & I & II & III & IV & Total \\
\hline & Cases (\%) & Cases $(\%)$ & Cases $(\%)$ & Cases (\%) & Cases $(\%)$ & Cases (\%) \\
\hline Radiation pneumonitis & $19(51.3)$ & $5(13.5)$ & $10(27.0)$ & $3(8.1)$ & $0(0)$ & $18(48.7)$ \\
\hline Radiation esophagitis & $8(21.6)$ & $10(27.0)$ & $14(37.8)$ & $5(13.5)$ & $0(0)$ & $29(78.4)$ \\
\hline Nausea & 7 (18.9) & $8(21.6)$ & $12(32.4)$ & $\begin{array}{ll}0 & (0)\end{array}$ & $0(0)$ & $30(81.1)$ \\
\hline Vomiting & $26(70.3)$ & $5(13.5)$ & $6(16.2)$ & $(0)$ & $0(0)$ & 11 (29.7) \\
\hline
\end{tabular}

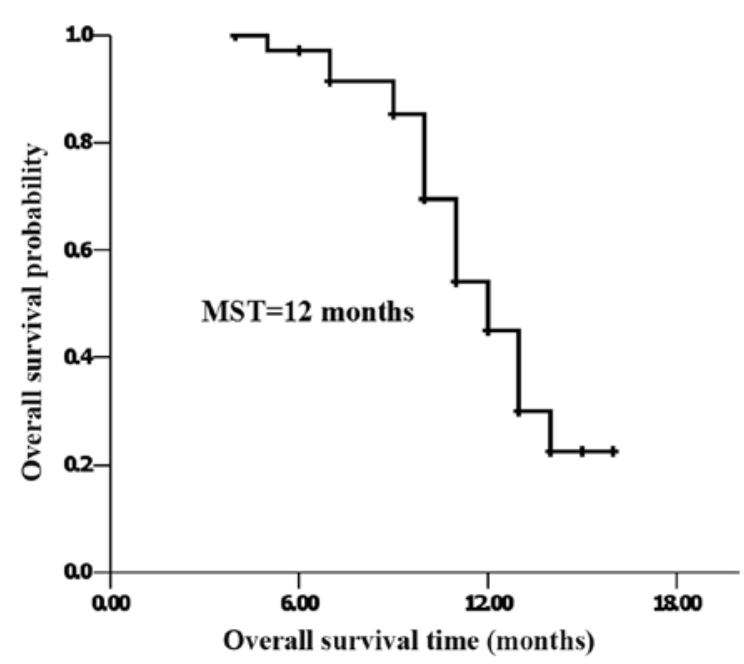

Figure 1. For all 37 eligible patients, the median survival time from treatment initiation was 12 months, whereas the 1-year overall survival rate was $45.5 \%$.

Hematological toxicity. Less severe hematological toxicity was observed despite its high incidence. Grade III/IV neutropenia occurred in $18.9 \%$ (7/37) of the patients, including 2 patients treated with antibiotics: Grade III/IV thrombocytopenia was observed in $8.1 \%$ (3/37) of patients, including one patient transfused with platelets; the incidence of anemia was found to be $32.4 \%$ (12/37), although no incidence was graded III or above, and no blood transfusion was required (Table II).

Non-hematological toxicity. Radiation pneumonitis occurred in $48.7 \%(18 / 37)$ of the patients including $8.1 \%(3 / 37)$ at Grade III. No cases were observed at Grade IV or above. The incidence of radiation esophagitis was $78.4 \%$ (29/37), with
$64.9 \%(24 / 37)$ at Grade I/II and $13.5 \%$ (5/37) at Grade III. No non-hematological toxicity of Grade IV or above was reported. Nausea was frequently observed in up to $81.1 \%(30 / 37)$ of cases, although generally to a lesser degree (Grades I/II), and no cases of Grade III or above occurred. Vomiting occurred in $29.7 \%(11 / 37)$ of cases, all of which were at Grade I/II with no cases at Grade III or above. No other associated severe toxicity was observed, such as radiation myelitis or radiation pericarditis (see Table III).

Short-term response rate. Complete response (CR) rate of the patients was $13.5 \%(5 / 37)$, partial response (PR) rate was $64.9 \%(24 / 37)$, stable disease (SD) was $10.8 \%$ (4/37), and the progressive disease (PD) rate was 10.8 (4/37). The short-term response rate (CR+PR) was $78.4 \%(29 / 37)$.

Survival. At the last follow-up, 17 patients had survived and 20 patients had succumbed to the disease. MST was 12 months, and the 1-year accumulated survival rate was $45.5 \%$ (Fig. 1).

\section{Discussion}

An increasing number of clinical trials have proven that concomitant radiochemotherapy is significantly superior to the sequencing modality in locally advanced unresectable NSCLC $(4,14)$, although toxicity increases. A lower dose of radiation (55-60 Gy) has therefore been used in standard radiochemotherapy regimens $(2,15,16)$. Fletcher has suggested that a radiation dose of 80-100 Gy is required to cure lung cancer (17), validated by stereotactic hypofractionated radiotherapy with the fact that a BED no less than 100 Gy achieved a better efficacy than a BED of less than 100 Gy (18). Therefore, the radiation dose should be escalated in the concomitant combination regimen to improve both the local control rate of the tumor and the survival rate (19). 
It is feasible to administer high radiation doses with 3DCRT in NSCLC, since the dose on healthy tissue can be precisely calculated in 3DCRT, thus providing better protection of normal tissues. Results of RTOG suggest that a 74 Gy dose is safe in radiation combined with concomitant chemotherapy in NSCLC (6). Due to the differences in physique, the American regimen of concomitant chemoradiation cannot be applied to Chinese patients. Our previous dose escalation research study in Chinese esophageal cancer patients showed that the tolerable dose for Chinese patients is significantly lower than that recommended by RTOG $(20,21)$. Due to the absence of reports on dose tolerance of concomitant radiochemotherapy in NSCLC in China, a prospective exploratory trial was necessary in order to evaluate the tolerance of a high-dose radiotherapy of 70 Gy with concomitant chemotherapy, as well as to determine an effective method to decrease the incidence of severe complications.

One of the key factors limiting dose escalation in concomitant radiochemotherapy is radiation pneumonitis. Findings of studies both in China and worldwide have suggested that concomitant radiochemotherapy is safe provided that the dose volume parameters are controlled under the conditions: V $20 \leq 30 \%$ and V $30 \leq 20 \%(6,8-10)$. Therefore, we limited the pulmonary radiation dose during the high-dose radiotherapy to prevent severe radiation pneumonitis.

Another severe treatment-associated toxicity in the NSCLC concomitant regimen is myeloid suppression. Neutropenia and thrombocytopenia not only lead to treatment interruption, but may also be life-threatening in severe cases. Moreover, the incidence of radiation pneumonitis may be increased in concomitant radiochemotherapy due to the sensitizing effect of chemotherapeutic drugs. Therefore, it is also crucial to select an appropriate chemotherapeutic regimen. CALGB9431 (22) analyzed the superiority by combining cisplatin with paclitaxel, gemcitabine and NVB, respectively, in concomitant radiochemotherapy. By comparing results, it was found that there was significantly less associated toxicity with NVB than with paclitaxel or gemcitabine with Grade III/IV neutropenia (27, 53 and $51 \%$, respectively), and notably fewer cases of vomiting and anorexia (16, 15,8\% and 22,27, $12 \%$, respectively). Therefore, an NP regimen has been more frequently adopted for the concomitant treatment in China and in other countries. Given the strong emesis inducibility of cisplatin and the inconvenient hydration required with high dosages, we substituted carboplatin with cisplatin to reduce those side effects.

In our small-sample exploratory study, patient tolerance of 3DCRT combined with concomitant NC chemotherapy was the primary endpoint. The results showed that all patients tolerated the 70-Gy high-dose radiotherapy well, with minor treatment-associated side effects. Incidence of severe toxicity was comparatively low as the involved field radiation was adopted without preventive radiation on the drainage area of lymph nodes (11). Grade III radiation pneumonitis occurred in only $8.1 \%$ (3/37) of patients, with no cases at Grade IV or above. Most cases of radiation esophagitis were Grade I/II; only $13.5 \%$ (5/37) were Grade III, and none were Grade IV or above. Severe nausea, emesis and anorexia were rare. Peripheral venous infusion was deemed necessary for only a few patients and was successful for a short period of time, with no need for nasal gastric feeding or parenteral hyperalimentation.

A Chinese study also adopted the concomitant NVB plus carboplatin regimen (NC) during radiotherapy in NSCLC. However, no 3DCRT, but rather conventional radiation technology was used in that study, and a preventive radiation treatment on ipsilateral pulmonary hilar and mediastinum was carried out. Despite the total dose being only $60 \mathrm{~Gy}$, the side effects were severe with Grade III radiation esophagitis in up to $42.6 \%$ of patients and Grade III radiation pneumonitis in up to $30.4 \%$ of patients (23). Another small-sample investigation on conventional radiation combined with concomitant NVB plus cisplatin (NP) reported a treatment-associated mortality rate of 3.8\% (1/26) due to esophageal-mediastinal fistula (27).

Although a relatively high radiation dose $(70 \mathrm{~Gy})$ was adopted in our study and the dose of concomitant chemotherapy remained constant throughout the study, the incidence of severe hematological toxicity was low, with $18.9 \%(7 / 37)$ of patients experiencing Grade III/IV neutropenia, 8.1\% (3/37) Grade III/IV thrombocytopenia and only one patient requiring platelet transfusion, with no cases of anemia at Grade III or above. However, a higher incidence of Grade III/IV neutropenia (35 and 33\%, respectively) was observed in other studies that combined radiation with concomitant NP $(25,26)$, which may be explained by the fact that the AUC adopted for carboplatin calculation in this study better satisfied the pharmacokinetics. Moreover, the incidence of severe radiation esophagitis of up to $25-30.8 \%$ in the previous concomitant NP study $(25,27,28)$ compared with $13.5 \%$ in the present study suggested that the preliminary advantage of concomitant $\mathrm{NC}$ during radiotherapy was that this type of radiotherapy is well-tolerated.

The short-term response rate of NC combined with concomitant radiotherapy was up to $78.4 \%$, which was comparative to the NP regimen $(24,25,27)$. However, the 1-year accumulated survival rate $(45.5 \%)$ was a little lower than the results of over $60 \%$ observed in the abovementioned studies. This discrepancy may be due to the different inclusion criteria; in the above three studies only stage III patients were recruited, whereas $35 \%$ of the patients in this study were stage IV.

In conlusion, a high dose of 70 Gy 3DCRT combined with concomitant chemotherapy of NVB plus carboplatin in the two lungs (V20 $\leq 30$ and V30 $\leq 20 \%$ ) was well tolerated and achieved a favorable short-term response rate, MST and 1-year accumulated survival rate. The regimen was feasible for Chinese patients with NSCLC. Further evaluation of this regimen in a prospective controlled phase II trial is ongoing.

\section{References}

1. Parkin DM, Bray F, Ferlay $\mathbf{J}$ and Pisani P: Global cancer statistics, 2002. CA Cancer J Clin 55: 74-108, 2005.

2. Dillman RO, Herndon J, Seagren SL, Eaton WL Jr and Green MR: Improved survival in stage III non-small-cell lung cancer: seven year follow-up of cancer and leukemia group B (CALGB) 8433 trial. J Natl Cancer Inst 88: 1210-1215, 1996.

3. Furuse K, Fukuoka M, Kawahara M, Nishikawa H, Takada Y, Kudoh S, Katagami N and Ariyoshi Y: Phase III study of concomitant versus sequential thoracic radiotherapy in combination with mitomycin, vindesine, and cisplatin in unresectable stage III non-small cell lung cancer. J Clin Oncol 17: 2692-2699, 1999.

4. Liang HY, Zhou H, Li XL, Yin ZH, Guan P and Zhou BS: Chemo-radiotherapy for advanced non-small cell lung cancer: concomitant or sequential? It's no longer the question: a systematic review. Int J Cancer 127: 718-728, 2010. 
5. Perez CA, Pajak TF, Rubin P, Simpson JR, Mohiuddin M, Brady LW, Perez-Tamayo R and Rotman M: Long-term observations of the patterns of failure in patients with unresectable non-oat cell carcinoma of the lung treated with definitive radiotherapy. Report by the Radiation Therapy Oncology Group. Cancer 59: 1874-1881, 1987.

6. Bradley J: A review of radiation dose escalation trials for non-small cell lung cancer within the Radiation Therapy Oncology Group. Semin Oncol 32: S111-S113, 2005.

7. Bradley JD, Moughan J, Graham MV, Byhardt R, Govindan R, Fowler J, Purdy JA, Michalski JM, Gore E and Choy H: A phase I/II radiation dose escalation study with concomitant chemotherapy for patients with inoperable stages I to III nonsmall-cell lung cancer: phase I results of RTOG 0117. Int J Radiat Oncol Biol Phys 77: 367-372, 2010.

8. Graham MV, Purdy JA, Emami B, Harms W, Bosch W, Lockett MA and Perez CA: Clinical dose-volume histogram analysis for pneumonitis after 3D treatment for non-small cell lung cancer (NSCLC). Int J Radiat Oncol Biol Phys 45: 323-329, 1999.

9. Wang S, Liao Z, Wei X, Liu HH, Tucker SL, Hu CS, Mohan R, Cox JD and Komaki R: Analysis of clinical and dosimetric factors associated with treatment-related pneumonitis (TRP) in patients with non-small-cell lung cancer (NSCLC) treated with concomitant chemotherapy and three-dimensional conformal radiotherapy (3D-CRT). Int $\mathrm{J}$ Radiat Oncol Biol Phys 66: 1399-1407, 2006.

10. Yang LQ, Wu LP, Chen LJ, Zhang RN, Gao YQ, Chen GY and Liang NQ: Value of V20 and V30 parametres for predicting acute radiation lung toxicity after 3D radiotherapy for lung cancer. Chin J Cancer Prev Treat (In Chinese) 15: 280-282, 2008.

11. Zhu GY, Xia TY, Wang LH, Gao XS, Wang JJ, Li AF, Zhang FQ, $\mathrm{Ma} \mathrm{L}, \mathrm{Li}$ YX and Xu B: Consensus and controversies on delineation of radiotherapy target volume for patients with non-small cell lung cancer. Chin J Radiat Oncol (In Chinese) 17: 432-436, 2008.

12. Trotti A, Byhardt R, Stetz J, Gwede C, Corn B, Fu K, Gunderson L, McCormick B, Morrisintegral M, Rich T, Shipley W and Curran W: Common toxicity criteria: version 2.0. An improved reference for grading the acute effects of cancer treatment: impact on radiotherapy. Int J Radiat Oncol Biol Phys 47: 13-47, 2000.

13. Sun Y and Zhou J (ed). Medical Oncology [M], 4th edition. People's Medical Publishing House (In Chinese), Beijing, pp106-107, 2004.

14. El-Sharouni SY, Kal HB, Battermann JJ and Schramel FM: Sequential versus concomitant chemo-radiotherapy in inoperable stage III non-small cell lung cancer. Anticancer Res 26: 495-505, 2006

15. Sause W, Kolesar P, Taylor S IV, Johnson D, Livingston R, Komaki R, Emami B, Curran W Jr, Byhardt R, Dar AR and Turrisi A III: Final results of phase III trial in regionally advanced unresectable non-small cell lung cancer: Radiation Therapy Oncology Group, Eastern Cooperative Oncology Group, and Southwest Oncology Group. Chest 117: 358-364, 2000 .
16. Schaake-Koning C, van den Bogaert W, Dalesio O, Festen J, Hoogenhout J, van Houtte P, Kirkpatrick A, Koolen M, Maat B and Nijs A: Effects of concomitant cisplatin and radiotherapy on inoperable non-small-cell lung cancer. N Engl J Med 326: 524-530, 1992.

17. Fletcher GH: Clinical dose response curves of human malignant epithelial tumors. Br J Radiol 46: 151, 1973.

18. Onishi H, Shirato H, Nagata Y, Hiraoka M, Fujino M, Gomi K, Niibe Y, Karasawa K, Hayakawa K, Takai Y, Kimura T, Takeda A, Ouchi A, Hareyama M, Kokubo M, Hara R, Itami J, Yamada K and Araki T: Hypofractionated stereotactic radiotherapy (HypoFXSRT) for stage I non-small cell lung cancer: updated results of 257 patients in a Japanese multi-institutional study. J Thorac Oncol 2: S94-S100, 2007.

19. Lichter AS and Lawrence TS: Recent advances in radiation oncology. N Engl J Med 332: 371-379, 1995.

20. Lin Q, Gao XS, Qiao XY, Zhou ZG, Zhang P and Yang XR: Dose escalation of cisplatin with 5-fluororacil in concomitant chemoradiotherapy for esophageal carcinoma. Chin J Radiat Oncol (In Chinese) 15: 301-304, 2006.

21. Lin Q, Gao XS, Qiao XY, Zhou ZG, Zhang P, Chen K, Zhao YN and Asaumi J: Phase I trial of escalating-dose cisplatin with 5-fluorouracil and concomitant radiotherapy in Chinese patients with esophageal cancer. Acta Med Okayama 62: 37-44, 2008

22. Vokes EE, Herndon JE II, Crawford J, Leopold KA, Perry MC, Miller AA and Green MR: Randomized phase II study of cisplatin with gemcitabine or paclitaxel or vinorelbine as induction chemotherapy followed by concomitant radiochemotherapy for stage IIIB non-small-cell lung cancer: cancer and leukemia group B study 9431. J Clin Oncol 20: 4191-4198, 2002.

23. Zhang ZM, Zhao HY, Zhang CJ and Mu JG: Vinorelbine and carboplatin with concomitant radiotherapy in locally advanced non-small cell lung cancer. Chin J Clin Oncol (In Chinese) 9: 625-627, 2004.

24. Rao CY: Study of concomitant versus sequential radiochemotherapy with vinorelbine and cisplatin in stage III non-small cell lung cancer. Chin J Cancer Prev Treat (In Chinese) 14: 942-943, 2007.

25. Liu J, Lv CX, Wang JM, Li HX, Guo JD, Wang CL, Gao LT and Zhao L: Analysis of two different concomitant radiochemotherapy regimens in the treatment of locally advanced stage III non-small cell lung cancer. Chin J Clin Oncol (In Chinese) 15: 226-229, 2010

26. Wang JP, Zhai XM, Zhang JN and Xu CS: Clinical study on concomitant radiotherapy in combination with vinorelbine and cisplatin for advance non-small cell lung cancer. Acta Med Jiangsu Univ (In Chinese) 16: 483-485, 2005.

27. Lu DJ, Wang L, Han C, Gao C and Li XN: Toxicity analysis for esophagus in locally advanced non-small cell lung cancer treatment using three dimensional conformal radiotherapy combined with concomitant chemotherapy. Chin J Radiol Med Prot (In Chinese) 30: 185-188, 2010

28. Zheng JX, Niu DL and Xu K: Radiotherapy combined with concomitant chemotherapy for stage III non-small cell lung cancer. Chin J Cancer Prev Treat (In Chinese) 13: 1822-1823, 2006. 